\title{
Six Sigma Methods and Formulas for Successful Quality Management
}

\author{
Dr. Maruti Prabhakar Rapaka \\ Ph.D., SSMBB, MCP, PRINCE 2, MSP, ITIL
}

ABSTRACT: This paper discusses the Methods of Six Sig ma and its applications used in project management. Keywor ds: Six Sig ma, Project Management, Standard Deviation, 3.4 defects, DMAIC, ANOVA.

\section{INTRODUCTION}

Six Sigma is basically the application of Statistical formulas and Methods to eliminate defects, variation in a product or a process. For example if you want to find the average height of male population in India, you cannot bring the entire population of more than 2 billion into one room and measure their height for a scenario like this we take samples that is we pick up sample(people) from each state and use statistical formulas to draw the inference about the average height of male population in a population which is more than 2 billion. One more example would be say a company manufactures pistons use $d$ in motor cycles the customer demand is that the piston should not a diameter more than $9 \mathrm{~cm}$ and less than $5 \mathrm{~cm}$ anything manufactured outside this limits is said to be a variation and the six sigma consultant should confirm that the pistons are manufactured within the said limits else if there is variation in the range then the company is not operating at 6 sigma level it is operating at a very low level.

A company is operating at six sigma level implies that there are only 3.4 defects per million opportunities for example an airline company operating at six sigma level means that it loses only 3.4 baggage's per million of the passenger it handles.

Below is Shown the Six Sigma Table and a graph explaining the meaning of various levels of Six Sigma.

\begin{tabular}{|l|l|l|}
\hline Sigma Level & Defect Rate & $\begin{array}{l}\text { Yield } \\
\text { Percentage }\end{array}$ \\
\hline $2 \sigma$ & $\begin{array}{l}308,770 \text { dpmo (Defects Per } \\
\text { Million Opportunities) }\end{array}$ & $69.10000 \%$ \\
\hline $3 \sigma$ & 66,811 dpmo & $93.330000 \%$ \\
\hline $4 \sigma$ & 6,210 dpmo & $99.38000 \%$ \\
\hline $5 \sigma$ & 233 dpmo & $99.97700 \%$ \\
\hline $6 \sigma$ & 3.44 dpmo & $99.99966 \%$ \\
\hline
\end{tabular}

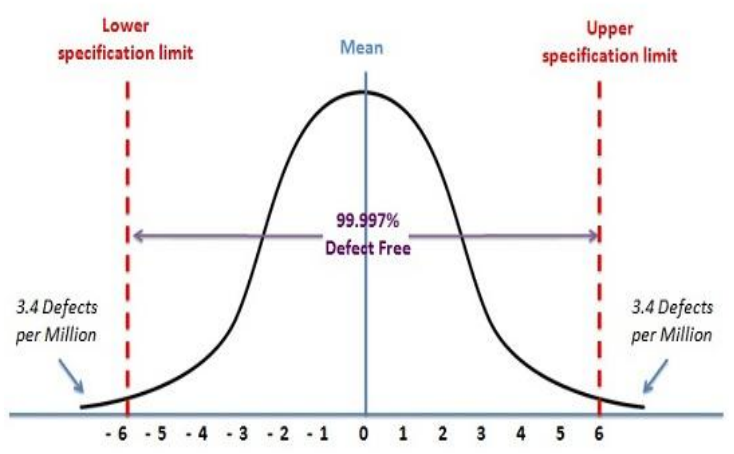

Six Sig ma is Denoted by the Greek alphabet $\sigma$ which is shown in the table above and is called as Standard deviation. The father of Six Sig ma is Bill Smith who coined the term Six Sigma and implemented it in Motorola in the 1980's.

Six Sigma is implemented in Five Phases which are Define, Measure, Analyze, Improve, Control and we will discuss each phases in brief and the various methods used in Six Sigma.

Define

The objectives within the Define Phase which is first phase in DMAIC framework of Six Sigma are: -

Define the Project Charter

- Define scope, objectives, and schedule

- Define the Process (top-level) and its stake holders

- Select team members

- Obtain Authorization from Sponsor

Assemble and train the team.

Project charters the charter documents the why, how, who and when of a project include the following elements

- Problem Statement

- Project objective or purpose, including the business need addressed

- Scope

- Deliverables

- Sponsor and stakeholder groups

- Team members 
- $\quad$ Project schedule (using GANTT or PERT as an attachment)

- Other resources required

Work break down Structure

It is a process for defining the final and intermediate products of a project and their relationship. Defining Project task is typically complex and accomplished by a series of decomposition followed by a series of aggregations it is also called top down approach and can be used in the Define phase of Six Sig ma framework.

Now we will get into the formulas of Six Sigma which is shown in the table below.

Central tendency is defined as the tendency for the values of a random variable to cluster round its mean, mode, or median.

\begin{tabular}{|l|l|l|}
\hline Central Tendency & Population & Sample \\
\hline Mean/Average & $\mu=\sum(\mathrm{Xi})^{\wedge} 2 / \mathrm{N}$ & $\begin{array}{l}\mathrm{XBar}=\sum \\
(\mathrm{Xi})^{\wedge} 2 / \mathrm{n}\end{array}$ \\
\hline Median & $\begin{array}{l}\text { Middle value of Data } \\
\text { Most occurring value in the data }\end{array}$ \\
\hline Mode & \multicolumn{1}{|c|}{} & \\
\hline Dispersion & $\begin{array}{l}\sigma 2 \\
\mu)^{\wedge} 2 / \mathrm{N}\end{array}$ & $\begin{array}{l}\mathrm{S}^{\wedge}=\sum\left(\mathrm{X}=\sum \mathrm{Xi}^{\wedge}\right. \\
\mathrm{XBar})^{\wedge} / \mathrm{n}-1\end{array}$ \\
\hline Variance & $\begin{array}{l}\sigma=\sqrt{ } \sum(\mathrm{Xi}-\mu)^{\wedge} 2 / \\
\mathrm{N}=\sqrt{ } \sum(\mathrm{Xi}- \\
\mathrm{XBar})^{\wedge} 2 / \mathrm{n}-1\end{array}$ \\
\hline StandardDeviation & $\mathrm{Max}-\mathrm{Min}$ \\
\hline Range & \multicolumn{2}{|l}{} \\
\hline
\end{tabular}

Where mean is the average for example if you have taken 10 sample of pistons randomly from the factory and measured their diameter the average would be sum of the diameter of the 10 pistons divided by 10 where 10 the number of observations the sum in statistics is denoted by $\sum$. In the above table $\mathrm{X}$, $\mathrm{Xi}$ are the measures of the diameter of the piston and $\mu, \mathrm{X}$-Bar is the average.

Mode is the most frequently observed measurement in the diameter of the piston that is if 2 pistons out 10 samples collected have the diameter as $6.3 \& 6.3$ then this is the mode of the sample and median is the midpoint of the observations of the diameter of the pis ton when arranged in sorted order.

From the example of the piston we find that the formulas of mean, median, mode does not correctly depict variation in the diameter of the piston manufactured by the factory but standard deviation formula helps us to find the variance in the diameter of the piston manufactured which is varying from the customer mentioned upper specification limit and lower specification limit.

The most important equation of Six Sigma is $Y=f(x)$ where $Y$ is the effect and $x$ are the causes so if you remove the causes you remove the effect of the defect. For example, headache is the effect and the causes are stress, eye strain, fever if you remove this causes automatically the headache is removed this is implemented in Six Sig ma by using the Fishbone or Ishikawa diagram invented by Dr Kaoru Ishikawa.
Measure Phase: In the Measure phase, we collect all the data as per the relationship to the voice of customer and relevantly analyze using statistical formulas as given in the above table. Capability analyses is done in measure phase.

The process capability is calculated using the formula CP = US L-LS L/6 * Standard De viation where $\mathrm{CP}=$ process capability index, USL = Upper Specification Limit and LSL $=$ Lower Specification Limit.

The Process capability measures indicates the following

1. Process is fully capable

2. Process could fail at any time

3. Process is not capable.

When the process is spread well within the customer specification the process is considered to be fully capable that means the $\mathrm{CP}$ is more than 2.In this case, the process standard deviation is so small that 6 times of the standard deviation with reference to the means is within the customer specification.

Example: The Specified limits for the diameter of car tires are $\mathbf{1 5 . 6}$ for the upper limit and $\mathbf{1 5}$ for the lower limit with a process mean of $\mathbf{1 5 . 3}$ and a standard de viation of 0.09 . FindCp and $\mathrm{Cr}$ what can we say about Process Capabilities?

$\mathrm{Cp}=\mathrm{USL}-\mathrm{LSL} 6 *$ Standard deviation $=15.6-15$

$/ 6 * 0.09=0.6 / 0.54=1.111$

$\mathrm{Cp}=1.111$

$\mathrm{Cr}=1 / 1.111=0.9$

Since $\mathrm{Cp}$ is greater than 1 and therefore $\mathrm{Cr}$ is less than 1; we can conclude that the process is potentially capable.

\section{Analyze Phase:}

In this Phase, we analyze all the data collected in the measure phase and find the cause of variation. Analyze phase use various tests like parametric tests where the mean and standard deviation of the sample is known and Nonparametric Tests where the data is categorical for example as Excellent, Good, bad etc.

Parametric Hypothesis Test -A hypothesis is a value judgment made about a circumstance, a statement made about a population. Based on experience an engineer can for instance assume that the amount of carbon monoxide emitted by a certain engine is twice the maximum allowed legally. However, his assertions can only be ascertained by conducting a test to compare the carbon monoxide generated by the engine with the legal require ments.

If the data used to make the comparison are parametric data that is data that can be used to derive the mean and the standard deviation, the 
population from which the data are taken are normally distributed they have equal variances. A standard error based hypothes is testing using the $\mathrm{t}$ test can be used to test the validity of the hypothesis made about the population. There are at least 3 steps to follow when conducting hypothesis.

1. Null Hypothesis: The first step consists of stating the null hypothesis which is the hypothesis being tested. In the case of the engineer making a statement about the level of carbon monoxide generated by the engine, the null hypothes is is

H0: the level of carbon monoxide generated by the engine is twice as great as the legally required amount. The Null hypothes is is denoted by $\mathrm{HO}$

2. Alternate hypothesis: the alternate (or alternative) hypothesis is the opposite of null hypothesis. It is assumed valid when the null hypothesis is rejected after testing. In the case of the engineer testing the carbon monoxide the alternative hypothesis would be

H1: The level of carbon monoxide generated by the engine is not twice as great as the legally required amount.

3. Testing the hypothesis: the objective of the test is to generate a sample test statistic that can be used to reject or fail to reject the null hypothesis. The test statistic is derived from $\mathrm{Z}$ formula if the samples are greater than 30 .

$\mathrm{Z}=\mathrm{Xbar}-\mu / \sigma / \sqrt{ } \mathrm{n}$

If the samples are less than 30 , then the t-test is used

$\mathrm{T}=\mathrm{X}$ bar $-\mu / \mathrm{s} / \sqrt{ } \mathrm{n}$ where $\mathrm{X}$ bar and $\mu$ is the mean and $\mathrm{s}$ is the standard deviation.

1-Sample t Test (Mean v/s Target) this test is used to compare the mean of a process with a target value such as an ideal goal to determine whether they differ it is often used to determine whether a process is off center

1 Sample Standard Deviation This test is used to compare the standard deviation of the process with a target value such as a benchmark whether they differ often used to evaluate how consistent a process is

2 Sample T (Comparing 2 Means) Two sets of different items are measured each under a different condition there the measurements of one sample are independent of the measurements of other sample.

Paired T The same set of items is measured under 2 different conditions therefore the 2 measurements of the same item are dependent or related to each other.

2-Sample Standard This test is used when comparing 2 standard deviations

Standard Deviation test This Test is used when comparing more than 2 standard deviations
Non-Parametric hypothesis Tests are conducted when data is categorical that is when the mean and standard deviation are not known examples are Chi-Square tests, Mann-Whitney U Test, Kruskal Wallis tests \& Moods Median Tests.

\section{Anova}

If for instance 3 sample means $\mathrm{A}, \mathrm{B}, \mathrm{C}$ are being compared using the t-test is cumbersome for this we can use analysis of variance ANOVA can be used instead of multiple t-tests.

ANOVA is a Hypothesis test used when more than 2 means are being compared.

If $\mathrm{K}$ Samples are being tested the null hypothesis will be in the form given below

$\mathrm{H} 0: \mu 1=\mu 2=\ldots . . \mu \mathrm{k}$

And the alternate hypothesis will be

H1: At least one sample mean is different from the others

If the data you are analyzing is not normal you have to make it normal using box cox transformation to remove any outliers (data not in sequence with the collected data).Box Cox Transformation can be done using the statistical software Minitab.

Improve Phase: In the Improve phase we focus on the optimization of the process after the causes are found in the analyze phase we use Design of experiments to remove the junk factors which don't contribute to smooth working of the process that is in the equation $Y=f(X)$ we select only the X's which contribute to the optimal working of the process.

Let us consider the example of an experimenter who is trying to optimize the production of organic foods. After screening to determine the factors that are significant for his experiment he narrows the main factors that affect the production of fruits to "light" and "water". He wants to optimize the time that it takes to produce the fruits. He defines optimum as the minimum time necessary to yield comestible fruits.

To conduct his experiment he runs several tests combining the two factors (water and light) at different levels. To minimize the cost of experiments he decides to use only 2 levels of the factors: high and low. In this case we will have two factors and two levels therefore the number of runs will be $2^{\wedge} 2=4$. After conducting observations he obtains the results tabulated in the table below.

\begin{tabular}{|l|l|}
\hline Factors & Response \\
\hline $\begin{array}{l}\text { Water -High Light } \\
\text { High }\end{array}$ & 10 days \\
\hline Water high - Light low & 20 days \\
\hline Water low - Light high & 15 days \\
\hline Water low - Light low & 25 days \\
\hline
\end{tabular}


Control Phase: In the Control phase we document all the activities done in all the previous phases and using control charts we monitor and control the phase just to check that our process doesn't go out of control. Control Charts are tools used in Minitab Software to keep a check on the variation. All the documentation are kept and archived in a safe place for future reference.

\section{CONCLUSION}

From the paper we come to understand that selection of a Six Sig ma Project is Critical because we have to know the long term gains in executing these projects and the activities done in each phase the basic building block is the define phase where the problem statement is captured and then in measure phase data is collected systematically against this problemstatement which is further analyzed in Analyze phase by performing various hypothes is tests and process optimization in Improve phase by removing the junk factors that is in the equation $y=f(x 1, x 2, x 3 \ldots \ldots)$ we remove the causes $x 1$, x2 etc. by the method of Design of Experiments and factorial methods. Finally we can sustain and maintain our process to the optimum by using control charts in Control Phase.

\section{REFERENCES}

[1] The Certified Master Black Belt Book By T. M Kubiak.

[2] The Six Sigma Hand Book by : Thomas Pyzdek and Paul Keller. 